Rapid Reviews COVID-19

\title{
Review 2: "Emergence of a novel SARS-CoV-2 strain in Southern California, USA"
}

François Balloux ${ }^{1}$

${ }^{1}$ UCL: University College London, Genetics, Evolution and Environment, UK

Published on: Feb 11, 2021

Dol: 10.1162/2e3983f5.f1ee2edd

License: Creative Commons Attribution 4.0 International License(CC-BY 4.0). 


\section{$\underline{\text { RR:C19 Evidence Scale rating by reviewer: }}$}

- Misleading. Serious flaws and errors in the methods and data render the study conclusions misinformative. The results and conclusions of the ideal study are at least as likely to conclude the opposite of its results and conclusions than agree. Decision-makers should not consider this evidence in any decision.

\section{Review:}

This very short paper (doi: https://doi.org/10.1101/2021.01.18.21249786) relates to the B.1.429 / CAL.20C lineage. It provides some background information and speculates that the spike in cases in California may have been caused by the higher transmissibility of the lineage.

The background is very sketchy and would have benefitted from being expanded on. In short, the first genome from that lineage was identified in Mexico on the 6th of July 2020 (GISAID accession number EPI_ISL_942929). At the time of writing this review, there have been 1298 sequences made available, with 873 from California. I'm aware of no evidence, including in the preprint that the surges in cases in California might have been caused by the higher transmissibility of this lineage.

The B.1429 is characterized by a combination of mutations (as all other lineages). Though, none of them looks overly concerning. The S protein L452R mutation marginally contributes to immune escape, though it lacks any of the more problematic mutations such as $\mathrm{E} 484 \mathrm{~K}$, or indels in the S1 domain. It also lacks the N501Y mutation which is a feature of three variants of concern.

In the absence of any functional evidence, I believe this lineage should not be considered as a 'variant of concern' and its local rise in frequency seems best explained by epidemiological stochasticity.

There are many lineages at reasonably high frequency in many places in the world. Some deserve our attention as they could be more transmissible, and others don't. Given the evidence provided in this preprint, I suspect that B 1.429 may be a fairly anonymous SARS-CoV-2 lineage.

Figure 1 is not a phylogenetic tree contrarily to its caption; I also didn't find it informative. Figure 2 is lifted as-is from NextStrain (https://nextstrain.org), without any acknowledgment to the original source. The submission lacks the acknowledgment table to contributors of genomes to the GISAID database.

To summarize, while I appreciate it is important to keep an eye on the B 1.429 lineage, the preprint does not provide any new meaningful evidence and would need significantly more work to be 
considered for publication. 